The University of San Francisco

USF Scholarship: a digital repository @ Gleeson Library |

Geschke Center

Kinesiology (Formerly Exercise and Sport Science)

College of Arts and Sciences

2008

\title{
Lower Limb Coordination and Stiffness During Landing from Volleyball Block Jumps
}

Gerwyn Hughes

University of San Francisco, ghughes@usfca.edu

James Watkins

Follow this and additional works at: https://repository.usfca.edu/ess

Part of the Sports Sciences Commons, and the Sports Studies Commons

\section{Recommended Citation}

Hughes, Gerwyn and Watkins, James, "Lower Limb Coordination and Stiffness During Landing from Volleyball Block Jumps" (2008). Kinesiology (Formerly Exercise and Sport Science). 37.

https://repository.usfca.edu/ess/37

This Article is brought to you for free and open access by the College of Arts and Sciences at USF Scholarship: a digital repository @ Gleeson Library | Geschke Center. It has been accepted for inclusion in Kinesiology (Formerly Exercise and Sport Science) by an authorized administrator of USF Scholarship: a digital repository@ Gleeson Library | Geschke Center. For more information, please contact repository@usfca.edu. 
Title: Lower limb coordination and stiffness during landing from volleyball block jumps.

Running head Landing coordination and stiffness

Authors: Gerwyn Hughes and James Watkins.

Institution: Department of Sports Science, Swansea University, Swansea, Wales.

Address: Gerwyn Hughes,

Department of Sports Science,

Swansea University,

Singleton Park,

Swansea,

Wales,

SA2 8PP.

e-mail: $\quad 189895 @$ swan.ac.uk

Telephone: $\quad$ +44 1792295086

Fax: $\quad$ +44 1792513171 


\begin{abstract}
.
The aim of the study was to investigate lower limb coordination and stiffness in five male and five female university volleyball players performing block jump landings. Coordination was assessed using angle - angle plots of the hip - knee, knee - ankle and hip - ankle joint couplings and discrete relative phase (DRP) of right - left joint couplings (i.e. left knee coupled with right knee). Leg stiffness was calculated as the ratio of the change in vertical ground reaction force (GRF) to the change in vertical displacement of the centre of gravity between ground contact and maximum vertical GRF. Knee stiffness was calculated as the ratio of the change in knee joint moment to the change in knee flexion angular displacement between ground contact and maximum knee joint moment. Comparison of the DRP angles between left and right legs indicated reduced symmetry between the left and right legs in females compared to males which may indicate greater likelihood of ligament strain in females compared to males. Furthermore, females exhibited reduced stability in the coordination between the left and right knee joints than males. Males exhibited significantly greater absolute and normalised leg stiffness and significantly greater absolute and normalised knee joint stiffness during landing compared to females. In conjunction with the coordination data, this may indicate reduced dynamic stability of the leg in females compared to males which may contribute to the greater incidence of ACL injury in females compared to males.
\end{abstract}

Key words: Coordination, stiffness, landing, ACL injury. 


\section{Introduction.}

Between $70 \%$ and $90 \%$ of anterior cruciate ligament (ACL) injuries have been reported to occur in non-contact situations (Griffin, Angel, Albohm, et al. 2000; McNair, Marshall, and Matherston 1993; Mykelbust, Maehlum, Engbretsen, et al. 1997). A non-contact situation is where there is no direct contact with the knee at the time of injury. Non-contact ACL injuries appear to occur most commonly during landing (Hume and Steele 1997; Otago and Neal 1997), rapid change of direction (Bartold 1997) and deceleration (Miller, Cooper, and Warner 1995). Furthermore, most non-contact ACL injuries appear to occur close to foot strike with the knee close to full extension and in a valgus position (Boden, Dean, Faegin, et al. 2000; Olsen, Mykelbust, Engebretsen, et al. 2004). Not surprisingly, the incidence of ACL injury is relatively high in sports involving a high frequency of landing, decelerating and rapid changes of direction such as basketball, netball, handball and volleyball (Arendt and Dick, 1995; Griffin, Angel, Albohm, et al. 2000). The incidence of non-contact ACL injury has been reported to be 6 to 8 times greater in females than in males competing in the same sports (Arendt and Dick 1995; Chandy and Grana 1985; Ferretti, Papandrea, Conteduca, et al. 1992; Gray, Taunton, McEnzie, et al. 1985; Gwinn, Wilkens, McDevitt, et al. 2000; Lindenfeld, Schmitt, Hendy, et al. 1994; Malone, Hardaker, Garrett, et al. 1993).

Previous studies (Griffin, Angel, Albohm, et al. 2000) have identified a number of possible intrinsic and extrinsic risk factors associated with the gender difference in the incidence of ACL injury. However, since the cause of ACL injury is likely to be the result of a complex interaction of risk factors (Lysens, Steverlynck, Van Den Auweele, et al. 1984), composite variables, including measures of coordination and stiffness, may provide more insight into the causes of ACL injury in general and the greater incidence of ACL injury in females in particular. 


\section{Coordination.}

A number of previous studies have examined gender differences in the angle - time histories of the lower limb joints during landing/cutting (Malinzak, Colby, Kikendall, et al. 2001; Decker, Torry, Wyland, et al. 2003; Hughes, Watkins, Owen, et al. 2007) which provide a measure of movement patterns of individual joints. The relative movement of one joint with respect to another, i.e. the coordination, may provide insight into the relationship between the lower limb joints and therefore the likely strain on the stabilising structures of the lower limb joints. However, there would appear to be no reports of lower limb coordination during landing/cutting manoeuvres in males and females. Coordination refers to the relative timing of motion between body segments (Jensen, Phillips, and Clark 1994). In any particular whole body movement, each body segment can be thought of as an independent oscillator that oscillates through a particular range of motion during the movement. The coordination between any two oscillators is the degree of coupling (relative timing or relative phasing) between these two oscillators. Since a landing manoeuvre is a discrete action, angle - angle plots are likely to provide the most appropriate illustration (qualitative) of relative motion, where the angle of one joint is plotted against the angle of another (Anderson and Sidaway 1994), and time series - based discrete relative phase (DRP) is likely to provide the most appropriate measure (quantitative) of relative timing between the hip, knee and ankle joints. DRP analysis determines the relative timing between key events in a movement cycle, such as the maximum angle of two different joints. 
Leg and knee joint stiffness.

Whilst analysis of the relative phase between pairs of joints provides an indication of the coordination between the joints, it is the coordination between all of the joints in the kinetic chain that determine the effectiveness and efficiency of the movement as a whole. In a landing manoeuvre, it is the coordination between the hip, knee and ankle joints that largely determine the dynamic stability of the leg. The dynamic stability of the leg is reflected in the stiffness of the leg, i.e. the resistance of the leg to compression (flexion of hip, knee and ankle joints) during landing. Leg stiffness refers to the stiffness of the leg when modelled as a single linear spring and is calculated as the ratio of the change in vertical GRF to the change in vertical displacement of the centre of gravity (CG) between ground contact and maximum vertical GRF (Farley and Morgenroth 1999). Knee joint stiffness refers to the torsional stiffness of the knee joint when modelled as a spring and is calculated as the ratio of the change in knee joint moment to the change in knee flexion angular displacement between ground contact and maximum knee joint moment (Farley and Morgenroth 1999).

Whilst studies have examined gender differences in leg stiffness during two-legged hopping (Granata, Padua, and Wilson 2002), to our knowledge no study has investigated gender differences in leg stiffness during tasks in which non-contact ACL injury is common, such as landing. Furthermore, previous studies (Granata, Padua, and Wilson 2002; Farley and Morgenroth 1999) only report absolute leg stiffness without normalising for body weight and height. Since leg stiffness is the combined effect of the stiffness in the hip, knee and ankle joints, the lower leg stiffness in females compared to males reported by Granata, Padua, and Wilson (2002) may be due, at least in part to reduced stiffness of one or more of the hip, knee and ankle joints in females compared to males. These differences in leg stiffness and possibly joint stiffness may indicate less dynamic stability of the legs in females compared to males 
which may in some way account for the greater incidence of ACL injury in females compared to males. To our knowledge, no study has examined gender differences in knee joint stiffness when performing tasks such as hopping, running or landing.

Aim.

The aim of the study was to compare lower limb coordination and stiffness in male and female university volleyball players performing block jump landings.

\section{Methodology.}

Subjects.

Five female (mean age $21.8 \pm 0.5$ years, mass $58.85 \pm 8.03 \mathrm{~kg}$ and height $1.66 \pm 0.08 \mathrm{~m}$ ) and five male (mean age $21.0 \pm 3.5$ years, mass $69.88 \pm 3.47 \mathrm{~kg}$ and height $1.76 \pm 0.10 \mathrm{~m}$ ) university volleyball players with between three and seven years experience of playing volleyball participated in the study. All subjects had no previous history of hip, knee or ankle injury and had not participated in any specific training programmes designed to reduce lower extremity injuries. Ethical approval was granted for the study by the University Ethics Committee and written consent forms were signed by all subjects prior to data collection.

Measurement system.

Ground reaction force (GRF) was measured using two AMTI force platforms embedded into the laboratory floor sampling at $600 \mathrm{~Hz}$. A 12 camera Vicon 512 system (Vicon, Oxford, England) sampling at $120 \mathrm{~Hz}$ was used to determine 3D coordinates of 39 retro-reflective markers (25 mm diameter). Markers were placed directly on each subject (on skin or on 
clothing covering the skin) in accordance with the Vicon system's plug-in gait marker set. All subjects wore tight fitting clothing in order to minimise movement of markers relative to the anatomical locations they were intended to designate. From the markers and anthropometric data (height, weight, leg length, knee width, ankle width, elbow width, wrist width and hand thickness) of each subject, the Vicon system calculated the 3D coordinates of the location of the whole body $\mathrm{CG}$ and the hip, knee and ankle angles in the sagittal plane. Knee joint moment was calculated as the equivalent to the muscle moment in the quasi static method of determining joint moment (equal and opposite to the external moment about the knee due to the ground reaction force) in the sagittal plane (Alexander and Vernon 1975). Based on a frequency content analysis of the 3D coordinate data, marker trajectories were filtered using a Woltring Filter with a low-pass cut-off frequency of $10 \mathrm{~Hz}$ and stop-band frequency of 30 Hz.

The laboratory was set up with a rope fixed horizontally to act as a volleyball net at a height of $2.43 \mathrm{~m}$ for male subjects and $2.24 \mathrm{~m}$ for female subjects. The net was placed $5 \mathrm{~cm}$ in front of and parallel to the adjacent force platforms. In addition to the net, a volleyball was suspended from the ceiling so that it was positioned $5 \mathrm{~cm}$ above the height of the net and with the centre of the ball $10 \mathrm{~cm}$ in front of the line of the net (the other side of the net to where the subject (blocker) was standing). The ball was positioned vertically above the line separating the two force platforms.

\section{Landing Task.}

The jumping and landing task was made as realistic as possible by having subjects attempt to block an actual spike performed by an experienced volleyball player. At the start of each trial, 
the subject stood with each foot on a separate force plate. The subject then timed his/her blocking action in order to try to block the ball as it was spiked. The ball was spiked from the same suspended position in order to eliminate variation in the position and velocity of the ball. On landing, each foot landed on a separate force plate. Following appropriate warm up and practice, data was recorded for three successful trials for each subject.

Angular definitions.

In the Plug-in gait system, the measurement of hip flexion/extension is based on the pelvic transverse axis (line connecting anterior superior iliac spine and posterior superior iliac spine markers) and the thigh axis (line connecting the hip joint and knee joint centres) projected onto the plane of hip flexion/extension (as determined by the plug-in gait marker system). The hip flexion/extension angle is the angle between the thigh axis and the line perpendicular to the pelvic transverse axis which passes through the hip joint centre. A positive angle corresponds to hip flexion (knee anterior to the trunk) relative to the fully extended position.

The measurement of knee flexion/extension is based on the thigh axis (line connecting the hip joint and knee joint centres) and the shank axis (line connecting the knee and ankle joint centres) projected onto the plane of knee flexion/extension (as determined by the plug-in gait marker system). The knee flexion/extension angle is the angle between the distal extension of the thigh axis and the shank axis. A positive angle corresponds to knee flexion relative to the fully extended position.

The measurement of ankle plantar/dorsiflexion is based on the foot axis (line connecting the ankle joint centre and the toe marker) and the shank axis (line connecting the knee and ankle 
joint centres) projected onto the plane of ankle dorsi/plantar flexion (as determined by the plug-in gait marker system). The ankle dorsi/plantar flexion angle is the angle between foot axis and a line perpendicular to the shank axis. A positive angle indicates dorsiflexion and a negative angle indicates plantarflexion.

From the angle - time histories of the hip, knee and ankle, DRP analysis was carried out to quantify coordination and angle - angle plots where used to illustrate coordination between hip - knee, knee - ankle and hip - ankle joint couplings. DRP angle was calculated between corresponding joints in the right and left leg, i.e., left knee coupled with right knee. The DRP angle was calculated using the following equation (Hamill, Haddad, and McDermott 2000):

DRP angle $\Phi=\frac{t_{2}-t_{1}}{t_{f}-t_{s}} \times 360^{\circ}$

where $t_{2}=$ time of maximum angle of left joint, $t_{l}=$ time of maximum angle of right joint, $t_{f}=$ time of zero velocity of the whole body CG and $t_{s}=$ time of initial ground contact (IC).

During a two-footed landing, the DRP angle between right and left lower limb joints indicates the symmetry between the right and left legs during landing which in turn may provide an indication of the symmetry in the loading of right and left lower limb joints. The DRP angle can range between $-360^{\circ}$ and $+360^{\circ}$, where $0^{\circ}$ indicates the timing of the two key events are perfectly in phase and an angle between $\pm 1^{\circ}$ and $\pm 360^{\circ}$ indicates the degree to which the timing of the two key events are out of phase. A positive DRP angle indicates that the joint in the left leg takes longer to reach its maximum angle than the corresponding joint in the right leg (e.g. left knee reaches its maximum angle after the right knee) and a negative DRP angle indicates that the joint in the right leg takes longer to reach its maximum 
angle than the corresponding joint in the left leg (e.g. right knee reaches its maximum angle after the left knee). The standard deviation of the DRP angle over a number of trials (vDRP) indicates the variability/stability in the coordination between right and left joints. A small vDRP angle indicates high stability in coordination whereas a large vDRP angle indicates low stability in coordination.

Whole body centre of gravity location.

The location of the whole body CG was determined by the Vicon system based on a 15 segment model defined by the plug-in gait marker set (right and left hand, right and left forearm, right and left upper arm, right and left foot, right and left shank, right and left thigh, head and neck, thorax, pelvis). The Vicon system incorporates the anthropometric data of Dempster (1955) as reported by Winter (1990).

Leg and knee stiffness calculations

Leg stiffness was calculated as the ratio of the change in vertical GRF to the vertical displacement of the whole body CG between IC and the maximum vertical GRF:

$$
k_{\text {leg }}=\frac{\Delta F}{\Delta L}
$$

where $k_{\text {leg }}=$ leg stiffness, $\Delta F=$ change in vertical GRF and $\Delta L=$ vertical displacement of the CG.

Knee joint stiffness was calculated as the ratio of the change in knee joint moment to the knee flexion angular displacement between IC and the maximum knee joint moment: 
$k_{\text {joint }}=\frac{\Delta M}{\Delta \theta}$

where $k_{\text {joint }}=$ knee joint stiffness, $\Delta M=$ change in knee joint moment and $\Delta \theta=$ knee flexion angular displacement.

Data analysis.

The angular displacement - time data were standardised with respect to average trial time (between IC and maximum knee flexion). Angular displacement mean data was based on 30 trials for males and 30 trials for females ( 5 subjects $\times 3$ trials $\times 2$ legs). Vertical GRF, vertical CG displacement and leg stiffness mean data was based on 15 trials for males and 15 trials for females (5 subjects $\times 3$ trials). Absolute and normalised (with respect to weight and height) leg stiffness data were calculated for males and females. Since the force-measurement system was unable to determine the centre of pressure on the left foot, knee joint moment data was only collected for the right leg. Therefore mean data was based on 15 trials for males and 15 trials for females $(5$ subjects $\times 3$ trials $\times 1 \mathrm{leg}$ ). Absolute and normalised (with respect to weight and height) knee joint stiffness data were calculated for males and females.

Independent-samples t-tests were carried out on the hip, knee and ankle angular displacement data (at initial ground contact, maximum flexion and range of motion), DRP angles for left right joint couplings, change in vertical GRF, vertical displacement of the CG, leg stiffness, change in knee joint moment, knee flexion angular displacement and knee joint stiffness to examine gender differences. Due to multiple t-tests (23) being carried out on samples taken from the same population, there was a high chance of statistical error due to multiple comparisons. Consequently, a Bonferroni adjustment to the alpha level was made. 


\section{Results.}

Angle - angle plots.

There was no significant difference in hip flexion at IC between males and females (Table 1), but maximum hip flexion was significantly greater in females than males. Consequently, the ROM of hip flexion was significantly greater in females than males. Knee flexion at IC was significantly greater in males than females (Table 1). There was no significant difference in the maximum knee flexion angle between males and females. However, the ROM of knee flexion was significantly greater in females than males. At IC, ankle plantarflexion was significantly greater in females compared to males (Table 1). There was no significant difference in the maximum angle of ankle dorsiflexion between males and females. However, the ROM of ankle plantar/dorsiflexion was significantly greater in females than males.

Table 1 about here.

The relationship between hip flexion and knee flexion, shown in Figure 1(a), was fairly linear (straight line on graph) throughout landing in males and females with males and females exhibiting greater knee flexion relative to hip flexion. Females exhibited a slightly greater rate of hip flexion relative to knee flexion than males, indicated by the peak gradient of the hip flexion - knee flexion coupling graph (Table 2).

The relationship between knee flexion and ankle plantar/dorsiflexion, shown in Figure 1(b), was fairly linear (straight line on graph) except at the end of the landing manoeuvre in males 
and females with males and females exhibiting greater ankle plantar/dorsiflexion relative to knee flexion. Males and females exhibited a similar rate of knee flexion relative to ankle plantar/dorsiflexion, indicated by the peak gradient of the knee flexion - ankle plantar/dorsiflexion coupling graph (Table 2).

The relationship between hip flexion and ankle plantar/dorsiflexion, shown in Figure 1(c), was fairly linear (straight line on graph) except at the end of the landing manoeuvre in males and females with males and females exhibiting greater ankle plantar/dorsiflexion relative to hip flexion. Males exhibited a greater rate of hip flexion relative to ankle plantar/dorsiflexion than females, indicated by the peak gradient of the hip flexion - ankle plantar/dorsiflexion coupling graph (Table 2).

Figure 1 about here.

Table 2 about here.

Discrete relative phase.

The lower the DRP angle between the two joints the tighter the coupling between the two joints (the closer the two events are in time in each cycle) and the lower the vDRP the more stable the coupling between the two joints. Table 3 shows the DRP angles and the vDRP angles for the left - right hip, knee and ankle joint couplings for males and females. The DRP angle for the left - right hip joint coupling in females was positive indicating that the right hip reached its maximum angle before the left hip. For the left - right joint couplings of the hips in males and the knees and ankles in both males and females, the DRP angle was 
negative indicating that the left joint reached its maximum angle before the right joint (Table $3)$.

Table 3 about here.

Whilst there were no significant differences between males and females, the DRP angles for the left - right joint couplings were greater for females than males for the hip, knee and ankle. The vDRP angle was greater for males than females for the hip and the ankle but the vDRP angle for the knee was greater for females than males. The DRP (symmetry) and the vDRP (stability) results indicate greater symmetry but lower stability between the left - right joint couplings in males compared to females.

Leg stiffness.

Both the absolute and normalised change in vertical GRF were significantly greater in males than females (Table 4). There was no significant difference in the absolute or normalised vertical displacement of the CG between males and females. However, both absolute and normalised leg stiffness were significantly greater in males than females.

Table 4 about here.

Knee stiffness.

There was no significant difference in the absolute or normalised change in knee joint moment between males and females (Table 4). Knee flexion angular displacement was 
significantly greater in males than females. Consequently, both absolute and normalised knee joint stiffness were significantly greater in males than females.

\section{Discussion.}

Coordination.

The angle - angle diagram of the hip - knee joint coupling (Figure 1a) shows that the knee moved through a greater angle relative to the hip during landing in both males and females. Peak gradient of the hip - knee curve was greater in females than males and range of motion of the hip and knee were greater in females than males (Tables 1 and 2).

The angle - angle diagram of the knee - ankle joint coupling (Figure 1b) shows that the ankle range of motion was greater relative to the knee during landing in both males and females but range of motion of knee flexion and ankle plantar/dorsiflexion was greater in females than males (Table 1). The peak gradients of the ankle - knee curves were similar for males and females (Table 2).

The angle - angle diagram of the hip - ankle joint coupling (Figure 1c) shows that the ankle range of motion was greater relative to the hip during landing in both males and females but range of motion of hip flexion and ankle plantar/dorsiflexion greater in females than males (Table 1). The peak gradients of the ankle - hip curves were similar for males and females (Table 2). Range of motion was significantly greater in all joints in females compared to males (Table 1). This increased range of motion in the hip, knee and ankle exhibited by females is consistent with the significantly lower leg stiffness and knee joint stiffness exhibited by females (Table 4) and may indicate less dynamic stability of the hip, knee and 
ankle joints in females compared to males. This may also be a protective mechanism where, through experience of playing volleyball, the female subjects used in the study have developed landing strategies which utilise a greater range of motion of the lower limb joints to reduce the ground reaction force acting on the body. This, in turn, is likely to reduce the strain on the support structures of the lower limb joints during landing.

During a two-footed landing, it is reasonable to assume that the DRP angle of the corresponding event in left and right legs (inter-limb joint coupling) should be very similar (i.e. the smaller the DRP angle for right - left hip, knee and ankle joints the greater the symmetry between the two legs). Comparison of the DRP angle between corresponding joints (hip, knee and ankle) in the right and left legs show a greater DRP angle in females for left right hip, knee and ankle joint couplings compared to males (Table 3). These results indicate less symmetry and, therefore, less coordination between the left and right legs during landing in females compared to males. The reduced symmetry in females compared to males may indicate greater asymmetry in loading on the passive support structures of the legs during landing in females compared to males.

The stability of the coordination (indicated by the vDRP angles) between left - right joint couplings was less for males than females for the hip and ankle joints but less for females than males for the knee joints (Table 3). The reduced stability in the coordination between the left and right knees in females compared to males may indicate less dynamic stability of the knees in females compared to males which may be a contributory factor in the gender difference in the incidence of non-contact ACL injury. To the authors' knowledge, no data has been reported on gender differences in lower limb coordination during landing, therefore 
comparison of the results of the present study with reference to previous research is not possible.

Leg stiffness.

Since the males weighed more than the females, it was not surprising that the change in absolute vertical GRF was significantly greater for males than females. However, even when normalised to body weight, the change in vertical GRF was still significantly greater in males than females. There was no significant difference between males and females absolute or normalised vertical displacement of the CG. However, the resulting absolute and normalised leg stiffness were significantly greater in males than females (Table 4). The reduced leg stiffness in females compared to males suggests reduced dynamic stability of the leg in females compared to males as females appear to be less able to resist compression of the leg during landing. This reduced dynamic stability of the leg in females compared to males may indicate increased likelihood of stain on the passive support structures of the knee in maintaining joint stability during landing in females compared to males which, in turn, may be a contributory factor in the increased incidence of non-contact ACL injury in females compared to males.

Granata, Padua, and Wilson (2002) reported leg stiffness to be significantly greater in males than females when hopping, supporting the findings of the present study. Mean values of leg stiffness reported by Granata, Padua, and Wilson (2002) were $33.9 \mathrm{kN} / \mathrm{m} \pm 4.2$ for males and $26.3 \mathrm{kN} / \mathrm{m} \pm 6.5$ for females. These values are greater than the values of leg stiffness observed for males $(15.02 \mathrm{kN} / \mathrm{m} \pm 8.82)$ and females $(10.29 \mathrm{kN} / \mathrm{m} \pm 3.56)$ in the present study. However, this is likely to be due to differences in the task; hopping involves storage 
and utilisation of strain energy and, therefore, maintenance of leg stiffness, whereas landing involves dissipation of strain energy and, therefore, a fairly rapid reduction in leg stiffness following the initial impact phase. Granata, Padua, and Wilson (2002) only reported absolute values for leg stiffness, therefore the gender difference in leg stiffness may have been due to differences in height and weight between males and females. However, the results of the present study suggest that even when normalised to weight and height, males still exhibit significantly greater leg stiffness than females.

Knee stiffness.

There was no significant difference in the absolute or normalised change in knee joint moment between males and females during landing. However, the knee flexion angular displacement was significantly greater in females than males. Consequently, the absolute and normalised knee joint stiffness was significantly greater in males than females during landing (Table 4). The reduced absolute and normalised knee stiffness in females compared to males may contribute to the reduced absolute and normalised leg stiffness in females compared to males. As the quadriceps and the hamstrings contract during landing, they act in a way to increase the joint contact forces and limit movement within the knee joint (maintain joint stability). The greater the ability of the muscles to resist rotation of the knee (i.e., the greater the knee joint stiffness) the greater the dynamic stability of the knee is likely to be and therefore the less likely the passive structures of the knee, such as the ACL, will be put under strain. Consequently, knee joint stiffness may be an important factor in preventing ACL injury. The reduced knee joint stiffness in females compared to males may indicate reduced dynamic stability of the knee during landing which may contribute, at least in part, to the greater incidence of non-contact ACL injury in females compared to males. To the authors' knowledge, no previous data has been reported for gender differences in absolute or 
normalised knee joint stiffness during landing, therefore comparison of the findings of the present study with reference to previous research is not possible.

The main limitation of the current study was the small sample size used. However, the results indicate potentially important findings for future research into the possible causes of the gender difference in the incidence of non-contact ACL injury. The results presented in this study provide data for variables not previously examined with regards to gender differences in landing biomechanics associated with ACL injury which may provide the basis for further study involving larger sample sizes.

\section{Summary and conclusions.}

Range of motion of hip, knee and ankle joints were greater for females than males during landing. Comparison of the DRP angle for left - right hip, knee and ankle joint couplings indicate less symmetry between the left and right legs during landing in females compared to males which may indicate greater asymmetry in loading on the passive support structures of the joints of the legs in females compared to males during landing which, in turn, may influence stain on the ACL. Furthermore, the reduced stability in the coordination between the left and right knee joints in female volleyball players compared to males during landing may indicate reduced dynamic stability of the knees during landing in females compared to males.

Males exhibited significantly greater absolute and normalised leg stiffness and significantly greater absolute and normalised knee joint stiffness during landing compared to females. In conjunction with the coordination data, this may indicate reduced dynamic stability of the 
legs in females compared to males. The reduced dynamic stability of the legs in female volleyball players compared to males during landing may contribute to the greater incidence of ACL injury in female volleyball players compared to males. Future research should examine gender differences in coordination and leg and knee joint stiffness in larger sample sizes and should investigate the effects of coordination and strength training on leg stiffness and knee joint stiffness in female volleyball players during landing. 
Acknowledgements.

No author has received any financial benefit for the research in this study. We have no potential conflicts of interest relevant to the contents of this manuscript. 


\section{Tables.}

Table 1. Group mean results for hip flexion, knee flexion and ankle plantar/dorsiflexion (plantarflexion; + dorsiflexion) angles at IC, maximum angle and ROM (Mean \pm standard deviation).

\begin{tabular}{lcccc}
\hline & & Hip flexion $\left(^{\mathbf{0}}\right)$ & Knee flexion $^{\mathbf{0}}$ ) & Ankle plantar/dorsiflexion $\left(^{\mathbf{0}}\right)$ \\
\hline \multirow{2}{*}{ Males } & IC & $13.89 \pm 5.78$ & $19.58 \pm 6.38^{1}$ & $-17.09 \pm 10.31^{2}$ \\
& Maximum & $29.16 \pm 7.91^{3}$ & $62.56 \pm 12.11$ & $31.39 \pm 6.51$ \\
& ROM & $15.27 \pm 9.29^{4}$ & $42.97 \pm 14.40^{5}$ & $48.49 \pm 12.28^{6}$ \\
\multirow{2}{*}{ Females } & Maximum & $39.07 \pm 11.89^{3}$ & $67.90 \pm 12.54$ & $-25.05 \pm 9.34^{2}$ \\
& ROM & $25.33 \pm 11.98^{4}$ & $53.13 \pm 13.75^{5}$ & $51.45 \pm 5.92$ \\
\hline
\end{tabular}

${ }^{1-6}$ : Significant difference between males and females $(\mathrm{p}<0.05)$. 
Table 2. Peak gradient of angle - angle coupling graphs.

\begin{tabular}{cccc}
\hline & Hip - knee & Knee - ankle & Hip - ankle \\
\hline Males & 0.38 & 1.43 & 4.12 \\
Females & 0.54 & 1.51 & 3.65 \\
\hline
\end{tabular}


Table 3. DRP and vDRP angles of left - right joint couplings for the hip, knee and ankle joints.

\begin{tabular}{cccc}
\hline & $\begin{array}{c}\text { Hip }\left(^{\mathbf{0}}\right) \\
\text { DRP } \pm \text { vDRP }\end{array}$ & $\begin{array}{c}\text { Knee }\left(^{\mathbf{0}}\right) \\
\text { DRP } \pm \text { vDRP }\end{array}$ & $\begin{array}{c}\text { Ankle }\left(^{\mathbf{0}}\right) \\
\text { DRP } \pm \text { vDRP }\end{array}$ \\
\hline Males & $-2.07 \pm 41.48$ & $-3.75 \pm 20.01$ & $-19.60 \pm 65.93$ \\
Females & $19.67 \pm 33.58$ & $-9.17 \pm 52.39$ & $-31.62 \pm 42.04$
\end{tabular}

* No significant differences between males and females. 
Table 4. Group mean results for change in vertical GRF, vertical displacement of the CG, leg stiffness, change in knee joint moment, angular displacement of the knee and knee stiffness during landing (mean \pm standard deviation).

\begin{tabular}{cccc}
\hline & & Males & Females \\
\hline Change in vertical & Absolute (N) & $2501.2 \pm 692.0^{1}$ & $1659.3 \pm 411.3^{1}$ \\
GRF & Normalised (BW) & $3.64 \pm 1.01^{2}$ & $2.87 \pm 0.60^{2}$ \\
\hline Vertical CG & Absolute (m) & $0.167 \pm 0.044$ & $0.161 \pm 0.048$ \\
displacement & Normalised (ht) & $0.095 \pm 0.025$ & $0.097 \pm 0.029$ \\
\hline Leg stiffness & Absolute (kN/m) & $15.02 \pm 8.82^{3}$ & $10.29 \pm 3.56^{3}$ \\
& Normalised (BW/ht) & $38.55 \pm 20.91^{4}$ & $29.61 \pm 7.94^{4}$ \\
\hline Change in knee joint & Absolute (N.m) & $121.6 \pm 76.6$ & $93.2 \pm 31.8$ \\
moment & Normalised (BW.ht) & $0.101 \pm 0.063$ & $0.097 \pm 0.033$ \\
displacement & Absolute $\left({ }^{\circ}\right)$ & $28.76 \pm 9.85^{5}$ & $51.90 \pm 14.17^{5}$ \\
\hline Knee stiffness & Absolute (N.m/ $\left.{ }^{\circ}\right)$ & $4.23 \pm 1.08^{6}$ & $1.79 \pm 0.90^{6}$ \\
& Normalised $\left(\mathrm{BW} . \mathrm{ht} /{ }^{\circ}\right)$ & $0.0035 \pm 0.0009^{7}$ & $0.0019 \pm 0.0009^{7}$ \\
\hline
\end{tabular}

${ }^{1-7}$ : Significant difference between males and females $(\mathrm{p}<0.05)$. 


\section{References.}

Alexander R, Vernon A (1975) The dimensions of knee and ankle muscles and the forces they exert. Journal of Human Movement Studies 1: 115-123.

Anderson DF, Sidaway B (1994) Coordination changes associated with practice of a soccer kick. Research Quarterly for Exercise and Sport 65: 93-99.

Arendt EA, Dick R (1995) Knee injury patterns among man and women in collegiate basketball and soccer. The American Journal of Sports Medicine 23: 694-701.

Bartold SJ (1997) Injury driven change to the fundamental design parameters of the Australian rules football boot. In: Abstracts of the Australian Conference of Science and Medicine in Sport; October 7-10 1997; Canberra. Bruce, (ACT): Sports Medicine Australia: $52-53$.

Boden BP, Dean GS, Faegin JA, et al. (2000) Mechanisms of anterior cruciate ligament injury. Orthopedics 23: 573-578.

Chandy TA, Grana WA (1985) Secondary school athletic injury in boys and girls: a threeyear comparison. Physician and Sports Medicine 13: 314-316.

Decker MJ, Torry MR, Wyland DJ, et al. (2003) Gender differences in lower extremity kinematics, kinetics and energy absorption during landing. Clinical Biomechanics 18: 662669.

Dempster WT (1955) Space requirements of the seated operator. WADC Technical report: 55-159, Wright-Patterson Air Force Base, Ohio.

Farley CT, Morgenroth DC (1999) Leg stiffness primarily depends on ankle stiffness during human hopping. Journal of Biomechanics 32: 267-273. 
Ferretti A, Papandrea P, Conteduca F, et al. (1992) Knee ligament injuries in volleyball players. The American Journal of Sports Medicine 20(2): 203-207.

Granata KP, Padua DA, Wilson SE (2002) Gender differences in active muscle stiffness. Part II. Quantification of leg stiffness during functional hopping tasks. Journal of Electromyography and Kinesiology 12(2): 127-135.

Gray J, Taunton JE, McEnzie DC, et al. (1985) A survey of injuries to the anterior cruciate ligament of the knee in female basketball players. International Journal of Sports Medicine 6: 314-316.

Griffin LY, Angel J, Albohm MJ, et al. (2000) Noncontact anterior cruciate ligament injuries: risk factors and prevention strategy. Journal of the American Academy of Orthopaedic Surgeons 8(3): 141-150.

Gwinn DE, Wilkens JH, McDevitt ER, et al. (2000) The relative incidence of anterior cruciate ligament injury in men and women at the United States naval academy. The American Journal of Sports Medicine 28: 98-102.

Hamill J, Haddad JM, McDermott WJ (2000) Issues quantifying variability from a dynamical systems perspective. Journal of Applied Biomechanics 16: 407-418.

Hughes G, Watkins J, Owen N, et al. (2007) Gender differences in knee kinematics during landing from volleyball block jumps. Human Movement Studies 53, 1-20.

Hume PA, Steele JR (1997) Injury prevention strategies in netball: are Australian players heeding the advice? In: Abstracts of the Australian Conference of Science and Medicine in Sport; October 7-10 1997; Canberra. Bruce, (ACT): Sports Medicine Australia, 306-307.

Jensen MT, Phillips SJ, Clark JE (1994) For young jumpers, differences are in movement's control, not in coordination. Research Quarterly for Exercise and Sport 65: 258-268. 
Lindenfeld TN, Schmitt DJ, Hendy MP, et al. (1994) Incidence of injury in indoor soccer. The American Journal of Sports Medicine 22(3): 354-371.

Lysens R, Steverlynck A, Van Den Auweele Y, et al. (1984) The predictability of sports injuries. Sports Medicine 1: 6-10.

Malinzak RA, Colby SM, Kikendall DT, et al. (2001) A comparison of knee joint motion patterns between men and women in selected athletic tasks. Clinical Biomechanics 16: 438445.

Malone TR, Hardaker WT, Garrett WE, et al. (1993) Relationship of gender to anterior cruciate ligament injuries in intercollegiate basketball players. Journal of the Southern Orthopaedic Association 2: 36-39.

McNair P, Marshall R, Matherston J (1993) Important features associated with acute anterior cruciate injury. The New Zealand Medical Journal 103: 537-539.

Miller MDM, Cooper DE, Warner JJP (1995) Review of sports medicine and arthroscopy. Philidelphia, PA: W.B. Saunders.

Mykelbust G, Maehlum S, Engbretsen L, et al. (1997) Registration of cruciate ligament injuries in Norwegian top level team handball: a prospective study covering two seasons. Scandinavian Journal of Medicine and Science in Sports 7: 289-292.

Olsen OE, Mykelbust G, Engebretsen L, et al. (2004) Injury mechanisms for anterior cruciate ligament injuries in team handball: A systematic video analysis. The American Journal of Sports Medicine 32(4): 1002-1012.

Otago L, Neal RJ (1997) Netball pivot landings- is there a correct way? In: Abstracts of the Australian Conference of Science and Medicine in Sport; October 7-10 1997; Canberra. Bruce, (ACT): Sports Medicine Australia, 262-263. 
Winter DA (1990) Biomechanics and motor control of human movement. New York, John Wiley. 


\section{Figure captions.}

Figure 1. Angle - angle diagrams of a) hip flexion $(\alpha)$ - knee flexion ( $\beta$ ) joint coupling, b) knee flexion $(\beta)$ - ankle plantar/dorsiflexion $(\gamma)$ joint coupling and c) hip flexion $(\alpha)-$ ankle plantar/dorsiflexion $(\gamma)$ between IC and maximum knee flexion for males and females. 